\title{
AC frequency characteristics of coplanar impedance sensors as design parameters
}

\author{
Jongin Hong, ${ }^{* a b}$ Dae Sung Yoon, ${ }^{* b}$ Sung Kwan Kim, ${ }^{a}$ Tae Song Kim, ${ }^{b}$ Sanghyo Kim, ${ }^{c}$ Eugene Y. Pak ${ }^{c}$ and \\ Kwangsoo No ${ }^{a}$
}

Received 7th July 2004, Accepted 2nd December 2004

First published as an Advance Article on the web 10th January 2005

DOI: $10.1039 / \mathrm{b} 410325 \mathrm{~d}$

\begin{abstract}
Glass-based microchannel chips were fabricated using photolithographic technology, and Pt thin-film microelectrodes, as coplanar impedance sensors, were integrated on them. Longitudinal design parameters, such as interelectrode spacing and electrode width, of coplanar impedance sensors were changed to determine AC frequency characteristics as design parameters. Through developing total impedance equations and modeling equivalent circuits, the dominant components in each frequency region were illustrated for coplanar impedance sensors and the measured results were compared with fitted values. As the ionic concentration increased, the value of the frequency-independent region decreased and cut-off frequencies increased. As the interelectrode spacing increased, cut-off frequencies decreased and total impedance increased. However, the width of each frequency-independent region was similar. As the electrode area increased, $f_{\text {low }}$ decreased but $f_{\text {high }}$ was fixed. We think that the decrease in $R_{\text {Sol }}$ dominated over the influence of other components, which resulted in heightening $f_{\text {low }}$ and $f_{\text {high }}$. The interelectrode spacing is a more significant parameter than the electrode area in the frequency characteristics of coplanar sensors. The deviation of experimentally obtained results from theoretically predicted values may result from the fringing effect of coplanar electrode structure and parasitic capacitance due to dielectric substrates. We suggest the guidelines of dominant components for sensing as design parameters.
\end{abstract}

\section{Introduction}

In the last decade, there has been a tremendous development in the field of miniaturization of chemical and biochemical sensor devices. Also, the concept of a "Micro Total Analysis System" ( $\mu$ TAS), where attempts are made to completely integrate (bio)chemical systems on silicon or glass substrates, has been envisioned as a new concept for analytical devices, ${ }^{1}$ because it is expected that miniaturization will improve the speed and reliability of the measurements and will dramatically reduce the sample volume and the system costs.

The detection method most commonly used for a microfluidic platform is laser induced fluorescence (LIF) providing exquisite sensitivity with detection limits approaching the single molecule level. ${ }^{2-4}$ LIF also requires analytes that either show intrinsic fluorescence or can be readily associated with labeling chromophores. However, this detection provides high sensitivity and is well characterized and developed. But it is not suitable for use with portable equipment and especially for use with the new generation of microscale analysis systems. In regard to overall miniaturization, a lot of work is currently focusing on electrochemical methods, such as voltametry, amperometry, and potentiometry. ${ }^{5-9}$ The attractive feature of electrochemical detection includes the simple instrumentation required to carry out detection and the favorable sensitivity and limits of detection it offers. Because the electrical signal is

*kairduck@kaist.ac.kr (Jongin Hong)

dsyoon@kist.re.kr (Dae Sung Yoon) directly converted into the electronic domain via simple electrodes, the overall system can be more compact and the goal of miniaturization can be driven further. However, for amperometric detection, the target material must be intrinsically electroactive, or if it is not, an electroactive species must be appended to the target molecules. For voltametric detection, the use of the three-electrode structure in a potentiostatic setup overcomes the problems of reference degeneration. For potentiometric detection, the problem of integrating a stable and reliable reference system remains the main obstacle. Although the conductivity sensor has a lack of intrinsic selectivity, the simplicity of the sensor and the absence of problems in integrating the reference system make the sensor more attractive than others.

Over the past few decades, there have been considerable attempts in biosensors based on the impedimetric detection of biomolecules (e.g. specific ions, DNA, proteins, cells, plasma membranes, DNA hybridization, antigen-antibody recognition, bacteria viability, cell attachment, etc.). ${ }^{10-28}$ Electrical impedance-based sensors rely on the impedance change produced by the measured quantity in a material including conductivity and dielectric constant or in the geometry of a circuit related to cell constant, and thus the change can be used for the sensing mechanism of biological species. However, most of the impedance sensors have not been fully integrated on a single device. The common design of impedance sensors is thin metal rod or wires as electrodes, immersed in a flowthrough cell. Even microfabricated sensors are immersed in a vessel containing fluids or have a separate fluidic system, or 
the samples are dropped over an open reservoir on the sensors. Recently, these sensors have been implemented on the microchip format, but the sensing mechanism is just limited to the change of conductivity. ${ }^{19,21,23-28}$ Also, few studies have been reported on the design rule of impedance-based biosensors in microfluidic platforms. This article describes the coplanar impedance biosensors provided for electrochemical impedance measurements of a sample placed in a microfabricated glass capillary. In particular, we discuss the alternating current (AC) frequency characteristics of these sensors as parameters for sensor design, such as interelectrode spacing and electrode area. We suggest the design rule as sensing mechanisms.

\section{Materials and methods}

\subsection{Device design and fabrication}

Fig. 1(a) shows the layout of the glass devices studied, which consisted of a capillary channel and coplanar impedance sensors. Coplanar electrodes can be easily patterned at very small dimensions yielding miniaturized, reproducible, and ultimately low cost devices. However, if a two-dimensional planar structure is used, the fringing effect due to non-uniform field distribution is not neglected. ${ }^{29}$ It is exclusively dependent on the electrode configuration. A schematic diagram of rectangular planar electrodes on the glass device is shown in Fig. 1(b). The direction in parallel with the electric field or current lines is termed longitudinal ( $x$-axis), and that perpendicular to the electric field or current lines is termed transversal ( $y$-axis). Design parameters are as follows: the interelectrode spacing $D$, the electrode width $W$, a maximum length in the longitudinal direction $L_{\mathrm{L}}$, a maximum length in the transversal direction $L_{\mathrm{T}}$. In a design situation, particularly for the miniaturization of sensors, the application imposes dimensional constraints on the device that has to be realized. The primary design constraint on this device is the longitudinal dimension, such as $D$ and $W$. However, the transversal dimension, $L_{T}$ is fixed as $100 \mu \mathrm{m}$ due to the dimension of a microchannel. Several combinations of microelectrodes are designed as the variation of the longitudinal dimension, $D(20$, 100 , and $500 \mu \mathrm{m})$ and $W(20,100$, and $500 \mu \mathrm{m})$. From the

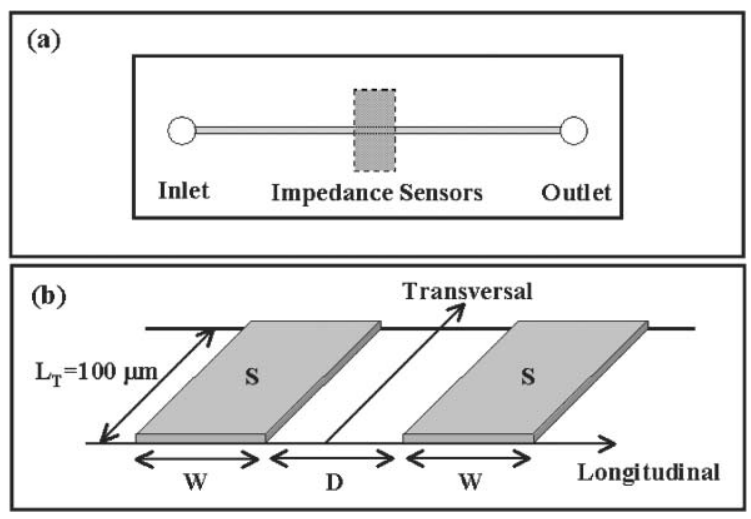

Fig. 1 Schematic diagram of a glass-based microchannel chip with coplanar impedance sensors: (a) layout of a glass device and (b) rectangular planar electrodes

experimental sets, we investigate the dependence of the AC frequency characteristics on design parameters in coplanar electrolytic impedance sensors.

Fig. 2 illustrates the microfabrication process using standard photolithographic technologies. ${ }^{30,31}$ Fabrication of the glassbased microchannel was as follows (Fig. 2(a)). Initially, the Corning 7740 Pyrex glass (Corning Co., New York, USA) wafer was cleaned with piranha solution $\left(\mathrm{H}_{2} \mathrm{SO}_{4}(\%): \mathrm{H}_{2} \mathrm{O}_{2}\right.$ $(\%)=3: 1)$ for $10 \mathrm{~min}$. It was then rinsed in deionized (DI) water and blown dry with nitrogen gas. The poly-silicon (200 nm) as etching mask for hydrofluoric acid (HF) etching was deposited on wafers using a low pressure chemical vapor deposition (LPCVD). Before photoresist (PR) coating, hexamethlysilane (HMDS) was used to improve PR adhesion. The wafer was coated with the AZ4620 PR (Clariant, Muttenz, Switzerland) using a spin coater at $4000 \mathrm{rpm}$ for $40 \mathrm{~s}$ and then baked on a hot plate at $95{ }^{\circ} \mathrm{C}$ for $2 \mathrm{~min}$. The UV lithography was processed using a mask aligner (EV620, Electronic Visions, Schaerding, Austria) with an exposure dose of $180 \mathrm{~mJ} \mathrm{~cm}{ }^{-2}$ for $12 \mathrm{~s}$. PR development was accomplished in $70 \mathrm{~s}$ by immersing the exposed substrate into a developer solution (AZ300MF, Clariant, Muttenz, Switzerland). The resulting $\mathrm{PR}$ patterns were then hard-baked in an oven at $110{ }^{\circ} \mathrm{C}$ for $1 \mathrm{~h}$ to harden the unexposed PR. The pattern was transferred into the poly-silicon using $\mathrm{CF}_{4}$ radio frequency (RF) plasma in reactive ion etching (RIE). After PR removal with acetone, the channel structures are etched into the glass using $49 \% \mathrm{HF}$ solution. The polysilicon layer was removed in the mixture of etchants $\left(\mathrm{HNO}_{3}: \mathrm{DI}: \mathrm{HF}=100: 40: 3\right)$. The resulting depth and width of the microchannel were $29.5 \mu \mathrm{m}$ and $100 \mu \mathrm{m}$, respectively. Holes for sample inlets, outlets, and

(a) Microchannel
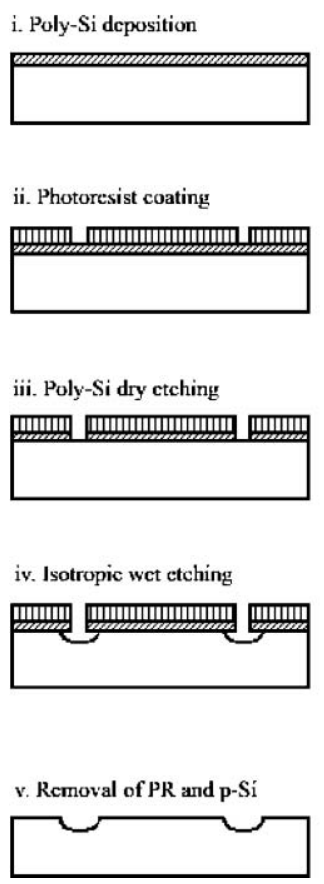

Fig. 2 Process flow for (a) microchannel etching and (b) microelectrode patterning. (b) Microelectrodes

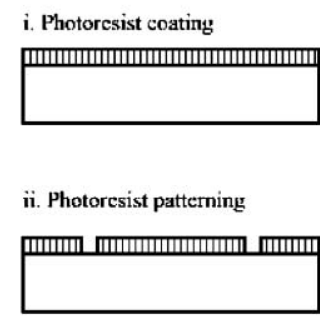

iii. Pt/li deposition

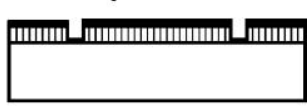

iv. Removal of PR
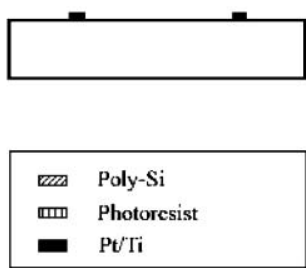
electrode contacts were drilled using sand blast methods (Fuel Cell Powers Co., Korea).

The lift-off sequence for coplanar impedance sensors was as follows (Fig. 2(b)). The image reversal process was performed to generate an undercut profile for lift-off. The glass wafer was coated with AZ1512 PR (Clariant, Muttenz, Switzerland) and then soft-baked on a hot plate at $95^{\circ} \mathrm{C}$ for $2 \mathrm{~min}$. The PR layer was exposed using EV620, and the wafer was baked on a hot plate at $110{ }^{\circ} \mathrm{C}$ for $3 \mathrm{~min}$. Then the patterned photoresist layer was developed in a developer solution. The Pt electrodes were fabricated by first sputtering $20 \mathrm{~nm}$ of Ti as the adhesion layer, followed by $200 \mathrm{~nm}$ of Pt (Inostech Co., Korea). The wafer was then immersed in acetone $(99.99 \%)$ to lift off the metal around the electrodes and rinsed in distilled $\mathrm{H}_{2} \mathrm{O}$. If necessary, ultrasonication was used to accelerate the process. Finally, both microchannel and impedance sensor wafers were fusionbonded at $550{ }^{\circ} \mathrm{C}$ for $30 \mathrm{~min}$ (BNP Co., Korea). Perfect sealing of the microchannels was observed (not shown in the figure), although electrodes protruding from one surface might interfere with glass-to-glass bonding. We sawed the finished wafer into an individual chip using an automatic dicing system.

\subsection{Reagents and detection apparatus}

The deionized water was provided from Promega Co. with $0.06 \mu \mathrm{S} \mathrm{cm}^{-1}$ of conductivity. The $1 \times \mathrm{TE}$ buffer solution from the Bioneer Co. was composed of $10 \mathrm{mM}$ of Tris- $\mathrm{HCl}$ and $1 \mathrm{mM}$ of ethylenediaminetetraacetic acid (EDTA). The TE buffer solution is usually used for capillary electrophoresis. Samples, such as $0.1 \times \mathrm{TE}$ and $1 \times \mathrm{TE}$, were prepared by the dilution of the $10 \times \mathrm{TE}$ buffer with the deionized water (DW). All solutions were filtered and degassed prior to use.

The impedance measurements were carried out using a Solartron 1260 frequency response analyzer (FRA) coupled to a 1296 dielectric interface (Solartron Analytical, Hamphire, UK). The Solartron 1260 was connected to an IEEE 488 GPIB interface on the personal computer. The 1296 dielectric interface enhances the capabilities of the 1260 FRA to cope with ultra low current at less than $100 \mu \mathrm{A}$ and capacitance levels at less than $1 \mathrm{pF}$. This combination can make impedance measurements, which exceed $100 \mathrm{~T} \Omega$. Also, the sample was screened to keep out the interference using a Faraday cage made in-house. The FRA can measure both the absolute value of impedance and phase shift with sweeping the frequency between $1 \mathrm{~Hz}$ and $10 \mathrm{MHz}$ with a $0.1 \mathrm{~V}$ (amplitude) voltage excitation. Specially developed Impedance software (Solartron Analytical, Hamphire, UK) was used to perform impedance measurements, and then the impedance data transferred to the Z-Plot/Z-View software package (Scribner Associates, Southern Pines, NC, USA). We can design equivalent circuit models to match physical characteristics of the measured cell using the Z-View, and determined the values which best fit our data based on a model we designed. When fitting, Z-View alters the values of the components and attempts to find the values which best fit your data. The type of data weighting is 'Calc-Modulus', which can give a better fit because each data points weight is normalized by its magnitude in covering a wide range of impedances. An alternate goodness of fit value is the weighted sum of squares (WSS) of the differences between the measured and calculated data, and this is particularly useful when comparing the WSS of fit of two different models to a single data set. The WSS becomes zero when the calculated data matches the experimental data exactly.

\section{Results and discussion}

\subsection{Theoretical approach}

A. Equivalent circuit models. Equivalent circuits are widely used to explain experimental impedance data in an equivalent manner, that is, they exhibit identical impedance for all frequencies. From the appropriate equivalent circuits, we can estimate the values of the circuit parameters, which reflect the carrier transport and the reaction in the electrolyte-electrode interface.

The current flowing between two electrodes is represented by the solution resistance, $R_{\mathrm{Sol}}$. If two electrodes of equal area $S\left(\mathrm{~m}^{2}\right)$ are placed in parallel at a perpendicular distance $D(\mathrm{~m})$ from each other, the solution resistance $R_{\text {Sol }}(\Omega)$, measured by driving the electrodes with an AC voltage $v(\mathrm{~V})$ and measuring the resulting current $i(\mathrm{~A})$, is related to the solution resistivity $\rho_{\text {Sol }}(\Omega \mathrm{m})$ by the equation

$$
R_{\mathrm{Sol}}=\frac{v}{i}=\rho_{\mathrm{Sol}} \frac{D}{S}
$$

Besides the $R_{\text {Sol }}$, a parasitic resistance, $R_{\text {Para }}$, is present in series with the sensor. This resulted from the resistance in the connecting cable, the bonding wires connected to the bondpads of the sensor and mainly Pt thin-film electrodes.

Direct capacitive coupling between electrodes is represented by the cell capacitance, $C_{\text {Cell }}$. The value of $C_{\text {Cell }}$ is determined by the dielectric constant of the electrolyte and the geometry of the electrodes. Generally, the equation of capacitance between two electrodes of a parallel plate capacitor is expressed as:

$$
C_{\text {Cell }}=\varepsilon_{r} \varepsilon_{0} \frac{S}{D}
$$

where $\varepsilon_{0}$ is the permittivity of free space, and has the value $8.854 \times 10^{-12} \mathrm{~F} \mathrm{~m}^{-1} . \varepsilon_{\mathrm{r}}$ is the relative dielectric constant of the medium between two electrodes. Additionally, a metal electrode in contact with an electrolyte possesses a natural charge density due to an excess or a deficiency of electrons at electrode-electrolyte interface. Charged particles from the electrolyte accumulate on the electrode in order to maintain electrical neutrality. This accumulation of ions forms an electrical double layer. The electrical double-layer consists of an adsorbed fixed layer, which is independent of electrolytic concentration, and a diffusive mobile layer, which is dependent of it. ${ }^{19,32-34}$ This electrostatic phenomenon is electrically represented by a double-layer capacitance, $C_{\mathrm{DL}}$. The total capacitance of the double-layer $\left(C_{\mathrm{DL}}\right)$ is made up of that due to the adsorption layer $\left(C_{\mathrm{Ads}}\right)$ and that due to the diffusive layer $\left(C_{\text {Diff }}\right)$, and these capacitances are connected in series:

$$
\frac{1}{C_{\mathrm{DL}}}=\frac{1}{C_{\mathrm{Ads}}}+\frac{1}{C_{\mathrm{Diff}}}
$$

If the electrolyte is very dilute, $C_{\mathrm{Ads}} \gg C_{\text {Diff }}$ and $C_{\mathrm{DL}} \approx C_{\text {Diff. }}$. On the other hand, when the solution is very concentrated, 
$C_{\text {Ads }} \ll C_{\text {Diff }}$ and $C_{\mathrm{DL}} \approx C_{\mathrm{Ads}} \cdot{ }^{19,21,32-34} C_{\mathrm{Ads}}$ is independent of the ionic concentration, while $C_{\text {Diff }}$ is a function of the ionic concentration.

From the consideration of possible electrical components mentioned above, equivalent circuits are modeled and shown in Fig. 3. The difference between two models is the component at the electrolyte-electrode interface: a $C_{\mathrm{DL}}$ in model I and a constant phase element (CPE) in model II. Actual systems show that the phase difference can be anywhere between $0^{\circ}$ and $90^{\circ}$ while still remaining constant over frequency. Thus, a better model for the interfacial response to AC signals is the CPE, which is given by an empirical formula: ${ }^{35,36}$

$$
Z_{\mathrm{CPE}}=T(j \omega)^{-P}
$$

where $T$ and $P$ are parameters that depend on the properties of the electrolytes and of the electrodes. $T$ is the inverse of the capacitance only when $P=1$. The equation assumes that the phase between voltage and current is constant. When the experimentally obtained results are fitted by commercially available software (Garmy, Scribner, Solartron, etc.), much better fit results can be obtained with CPE in comparison with the fit results obtained using the pure capacitor. The main problem in the use of commercially available software with CPE is the fact that capacity, obtained by the fitting procedure, does not have the dimension of capacity, i.e., $\left[\mathrm{F} \mathrm{cm}^{-2}\right]$, or $\left[\Omega^{-1} \mathrm{~cm}^{-2} \mathrm{~s}\right]$, but its dimension is given in $\left[\Omega^{-1} \mathrm{~cm}^{-2} \mathrm{~s}^{\alpha}\right]$ where $\alpha$ is the exponent in the equation for the CPE. The fit results using model II (WSS $<0.1$ ) were much better than that using model I (WSS < 5), so the CPE at the electrolyte-electrode interface was suitable to understand real systems. Therefore, we think that model I can be meaningful for developing the impedance equations because of the simplification of real systems, and model II can be useful for the phenomenological interpretation of real systems.

B. Frequency characteristics. Based on the impedance equation of the model I circuit shown in Fig. 3(a), the total
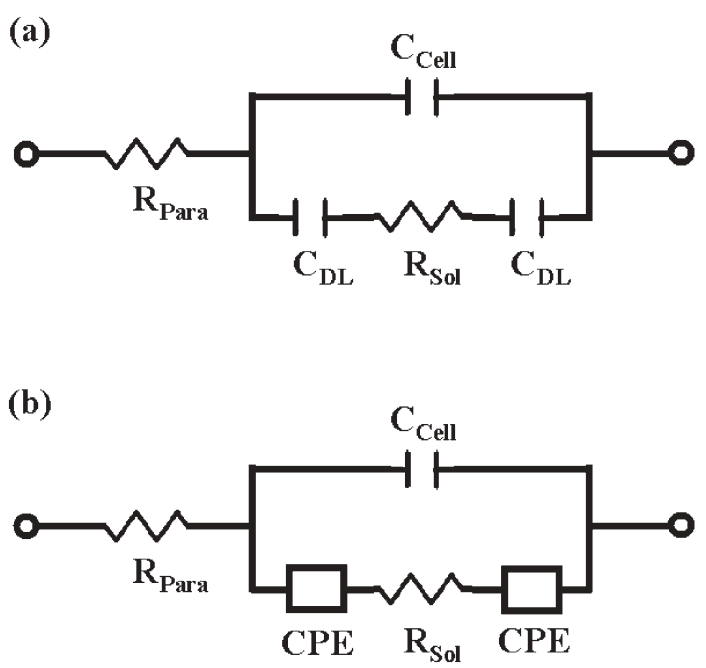

Fig. 3 Equivalent circuits to interpret impedance-frequency plots: (a) model I and (b) model II. impedance can be expressed as a function of the known values of the components and the frequency: ${ }^{19}$

$$
Z(j \omega)=2 R_{\text {Para }}+\frac{x}{j \omega C_{\mathrm{Cell}} x+1}, \quad x=R_{\mathrm{Sol}}+\frac{2}{j \omega C_{\mathrm{DL}}}
$$

At lower frequencies the impedance will be dominated by the $C_{\text {DL }}$ until the impedance of this capacitor becomes lower than the $R_{\text {Sol }}$. Then the sensor impedance becomes frequency independent. The low cut-off frequency can be expressed as eqn. (6), and is a function of the solution resistance and the double layer capacitance.

$f_{\text {low }} \approx \frac{1}{2 \pi\left(2 R_{\mathrm{Para}} C_{\mathrm{Cell}}+R_{\mathrm{Para}} C_{\mathrm{DL}}+\frac{1}{2} R_{\mathrm{Sol}} C_{\mathrm{DL}}\right)} \approx \frac{1}{R_{\mathrm{Sol}} C_{\mathrm{DL}}}$

However, at a certain frequency the impedance of the $C_{\text {Cell }}$ becomes lower than the $R_{\mathrm{Sol}}$. Then the impedance will decrease with an increase in frequency. The high cut-off frequency can be expressed as eqn. (7), and is a function of the solution resistance and the cell capacitance.

$$
f_{\mathrm{high}} \approx \frac{1}{2 \pi R_{\mathrm{Sol}} \frac{C_{\mathrm{DL}} C_{\mathrm{Cell}}}{C_{\mathrm{DL}}+2 C_{\mathrm{Cell}}}} \approx \frac{1}{R_{\mathrm{Sol}} C_{\mathrm{Cell}}}
$$

A schematic diagram of total impedance is shown in Fig. 4. There are three regions in the impedance spectrum, which correspond to the three types of components in equivalent circuit. The frequency-dependent property of these regions can be interpreted using the equivalent circuit mentioned above. As shown in Fig. 3 , there are two parallel branches $\left(C_{\text {Cell }}\right.$ and $C_{\mathrm{DL}}($ or $\mathrm{CPE})+R_{\mathrm{Sol}}+C_{\mathrm{DL}}$ (or CPE)). When the frequency is not sufficiently higher than $f_{\text {high }}$, the current cannot pass through the dielectric capacitor. That is, the capacitor is inactive, and just acts as an open circuit. Only the double layer capacitance and solution resistance in series are taken into account for the total impedance. Although both $C_{\mathrm{DL}}$ and $R_{\mathrm{Sol}}$ contribute to the total impedance below $f_{\text {high }}$, each of them dominates at different frequencies. The $C_{\mathrm{DL}}$ becomes essentially resistive at the frequency lower than $f_{\text {low }}$, and it offers a main contribution to the total impedance value. The impedance increases with the decrease in the frequency (double layer region). However, above $f_{\text {low }}$, double layer capacitance offers no impedance. Because the only solution resistance contributes to the impedance, the total impedance is independent of the frequency (solution resistance region). When the

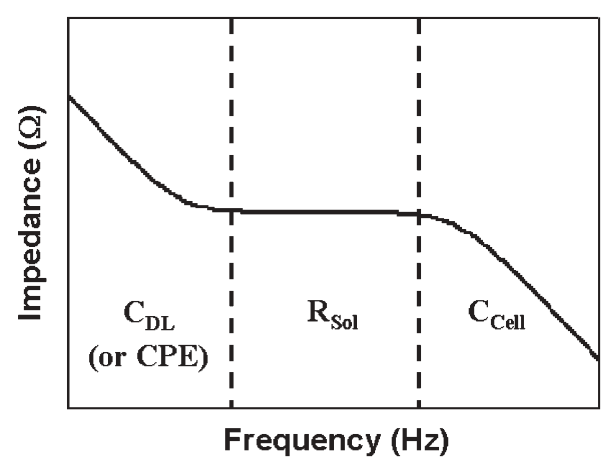

Fig. 4 Schematic diagram of total impedance-frequency plots divided into dominant components. 


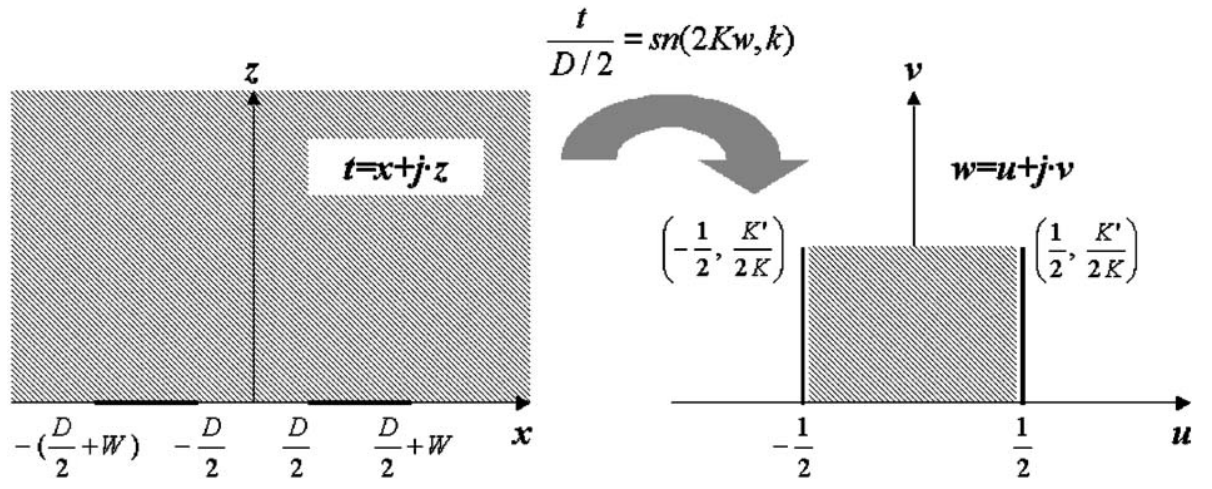

Fig. 5 Basic conformal transformation used to map semi-infinite half-plane into bonded rectangular region.

frequency is higher than $f_{\text {high, }}$, the current passes through the dielectric capacitor instead of the electrolyte solution resistance. That is, the branch $\left(C_{\mathrm{DL}}+R_{\mathrm{Sol}}+C_{\mathrm{DL}}\right)$ is inactive, and the branch $\left(C_{\text {Cell }}\right)$ is active. In this region, the dielectric capacitance of the medium dominates the total impedance, and the double layer capacitance and medium resistance can be ignored. Thus, the total impedance value is inversely proportional to the frequency.

C. Cell constant. The cell constant, which depends on the geometry of the sensor, has been well developed using the theory of conformal transformations. ${ }^{29,37}$ With this method each particular curve or shape on the real-world $(x, y)$ plane is converted into a corresponding curve or shape on the $(u, v)$ plane. The assumption is that the solution in the rectangular region (homogeneous medium) is given by a uniform distribution. The resistive cell constant per unit transversal length is defined as the proportionality factor between the measured resistance and the resistivity, while the capacitive cell constant per unit transversal length is done as the proportionality factor between the measured capacitance and the dielectric constant (Fig. 5).

$$
\begin{gathered}
K_{\text {Cell }}^{\mathrm{R}}=\frac{R_{\mathrm{Sol}}}{\rho} L_{\mathrm{T}}=\frac{2 K(k)}{K^{\prime}(k)} \\
K_{\text {Cell }}^{\mathrm{C}}=\frac{C_{\text {Cell }}}{\varepsilon_{0} \varepsilon_{\mathrm{r}} L_{\mathrm{T}}}=\frac{K^{\prime}(k)}{2 K(k)}
\end{gathered}
$$

where the modulus $k$ is related to the electrode lay-out, $K(k)$ is the complete integral of the complete integral of the first kind, and $K^{\prime}(k)$ is the complete integral of the first kind taken in the complementary modulus $k^{\prime}$.

$$
\begin{gathered}
k=\frac{D}{D+2 W} \\
K(k)=\int_{0}^{1} \frac{\mathrm{d} t}{\sqrt{\left(1-t^{2}\right)\left(1-k^{2} t^{2}\right)}} \\
K^{\prime}(k)=K\left(k^{\prime}\right) \quad k^{\prime}=\sqrt{1-k^{2}}
\end{gathered}
$$

Both resistive and capacitive cell constants as a function of $D$ and $W$ were calculated using DATAPLOT ${ }^{(i m}$ software (National Institute of Standards and Technology). The measured cell constant was calculated based on the highfrequency value of $R_{\mathrm{Sol}}$ and $C_{\text {Cell }}$. We compared the measured values with the theoretically obtained ones as design parameters.

\subsection{Experiments and analysis}

A. Electrolytic concentration. Fig. 6 shows Bode plots of total impedance against frequency for various TE buffers, where $D$ and $W$ are $100 \mu \mathrm{m}$ and $20 \mu \mathrm{m}$, respectively. Values of $C_{\text {Cell }}$ and $R_{\text {Sol }}$ were extracted from fitting in the high-frequency region, and then they were fixed when fitting using model II in all range of frequencies. The fitting results are summarized in Table 1. As the ionic concentration increased, $R_{\text {Sol }}$ decreased markedly but $C_{\text {Cell }}$ and CPE-T increased. The impedance sensors showed the linearity at the measuring frequencyindependent region (not shown in figure). If the sensitivity is defined as the ratio of solution resistivity $(\Omega \mathrm{m})$ to solution resistance $(\Omega)$, it was about $60\left(\mathrm{~m}^{-1}\right)$ in this sensor. Having CPE-P close to 1.0 for all samples indicates that the interface is mostly capacitive, with small parallel resistive component. We observed the horizontal resistive behavior, which was independent of frequency, and the points called cut-off frequencies, which deviate from the horizontal plateau. As the ionic concentration increased, the value of plateau decreased and the cut-off frequencies increased. These are related to the decrease

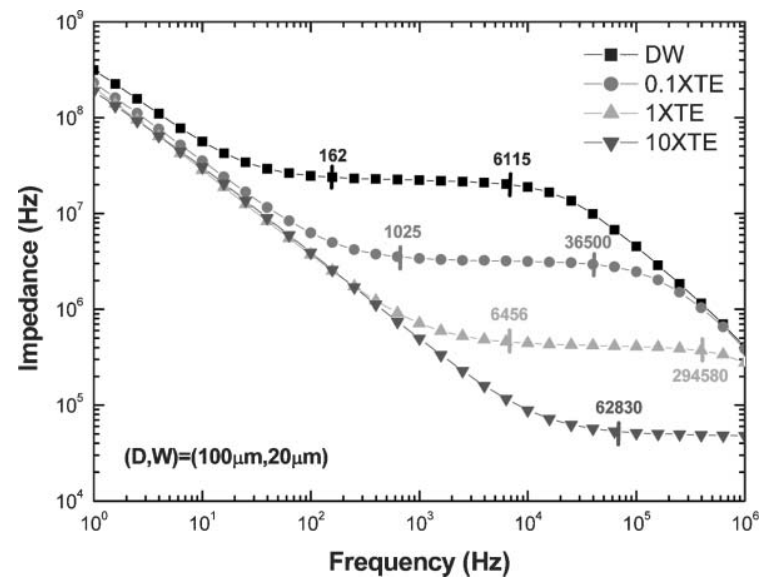

Fig. 6 Bode plots of total impedance against frequency for various TE buffers $(D=100 \mu \mathrm{m}$ and $W=20 \mu \mathrm{m})$. 
Table 1 Parameters for the circuit elements evaluated by fitting the impedance data using model II $(D=100 \mu \mathrm{m}, W=20 \mu \mathrm{m})$

\begin{tabular}{llrrr}
\hline & $C_{\text {Cell }} / \mathrm{pF}$ & $R_{\text {Sol }} / \mathrm{M} \Omega$ & CPE-T/pF & CPE-P \\
\hline Air & $0.295 \pm 0.005$ & & & WSS \\
DW & $0.355 \pm 0.007$ & $21.0 \pm 0.624$ & $591 \pm 25.2$ & $10^{-20}$ \\
$0.1 \times$ TE & $0.361 \pm 0.010$ & $3.08 \pm 0.067$ & $865 \pm 22.7$ & $0.845 \pm 0.010$ \\
$1 \times$ TE & $0.403 \pm 0.027$ & $0.412 \pm 0.010$ & $938 \pm 24.0$ & $0.849 \pm 0.005$ \\
\hline
\end{tabular}

in $R_{\text {Sol }}$ resulting from an increase in electrolyte conductivity. Because the decrease in $R_{\text {Sol }}$ dominates over the influence of the increase in $C_{\mathrm{DL}}$ and $C_{\mathrm{Cell}}$ on the cut-off frequencies, both $f_{\text {low }}$ and $f_{\text {high }}$ are heightened. Thus, the theoretical expectations correspond reasonably well with the experimental results of coplanar impedance sensors.

(a)

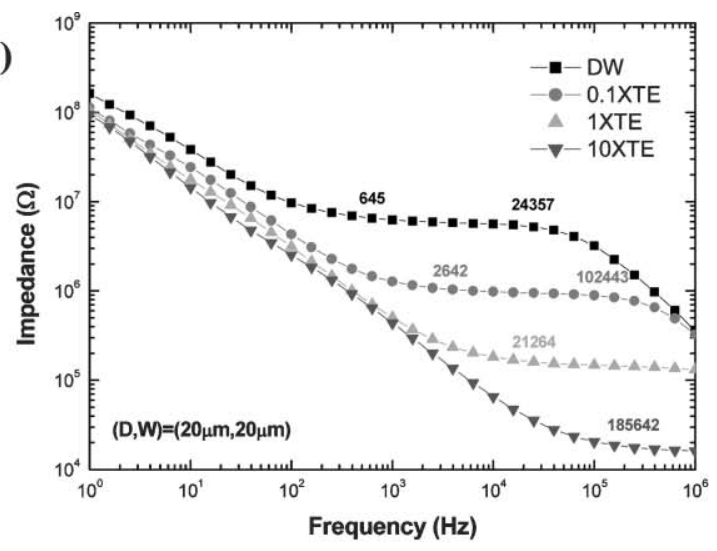

(b)

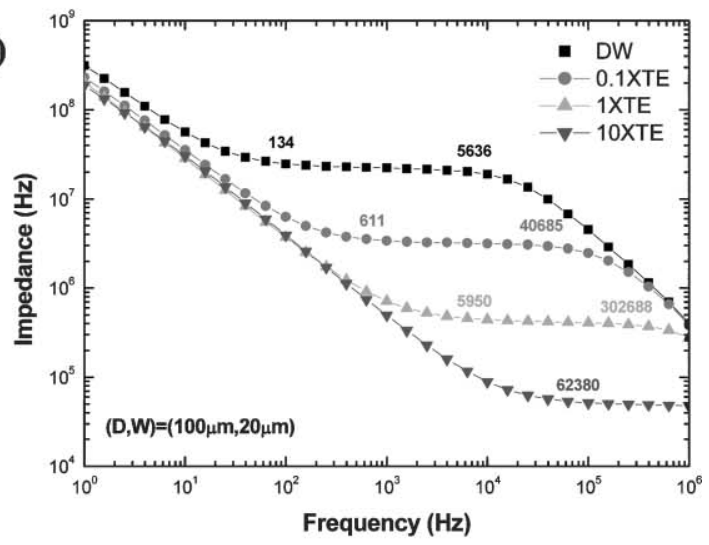

(c)

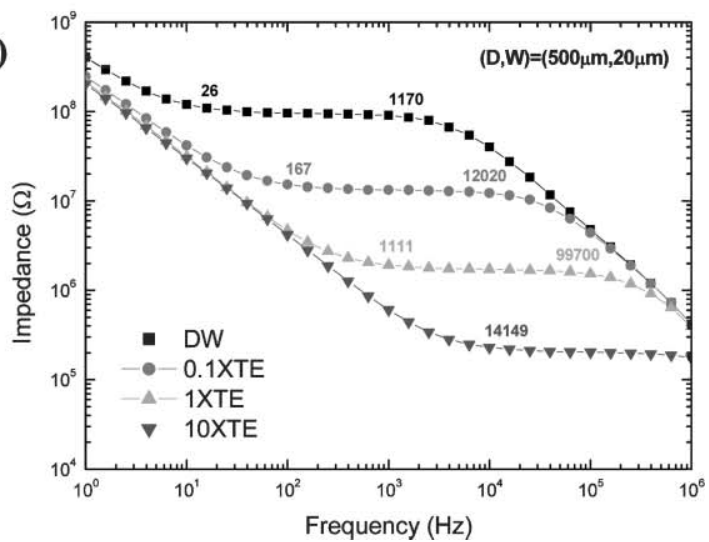

Fig. 7 Bode plots of total impedance against frequency as the variation in the interelectrode spacing ( $W=20 \mu \mathrm{m})$.
B. Interelectrode spacing. Because the transversal dimension of coplanar impedance sensors is restricted by the width of microchannel, their frequency characteristics must be evaluated as longitudinal constraints for the optimal sensor design. Fig. 7 shows Bode plots of total impedance against frequency as the variation in the interelectrode spacing. We observed that, as the spacing increased, the cut-off frequencies decreased but total impedance increased. The frequencyindependent region was not changed as the spacing so much. Fitting results, $C_{\text {Cell }}$ and $R_{\text {Sol }}$, as a function of interelectrode spacing $(W=20 \mu \mathrm{m})$ are summarized in Table 2. As the interelectrode spacing increased, $R_{\text {Sol }}$ increased but $C_{\text {Cell }}$ decreased slightly. $R_{\text {Sol }}$ was more dependent on the change of the spacing than $C_{\text {Cell }}$. To evaluate the capacitive component at the solution-electrode interface, we extracted data of capacitive magnitude at $10 \mathrm{~Hz}$ and phase using the simulation of CPE component. They are summarized in Table 3. The magnitude decreased slightly with an increase in the spacing. From the data of phase angle, the element at the interface can be represented as a leaky capacitor. The increase in $R_{\text {Sol }}$ resulted from the decrease in both low and high cut-off frequencies. Analogously, the change of $R_{\text {Sol }}$ overwhelmed that of the capacitive components, such as $C_{\mathrm{Cell}}$ and $C_{\mathrm{DL}}$.

The comparisons between the experimentally obtained cell constants and the theoretically predicted values are given in Fig. 8. Theoretically, as the interelectrode distance increases, the resistive cell constant increases but the capacitive one decreases. We observed that the steep rise of measured resistive cell constants with the increase in $D$. Although the difference between measured and predicted ones in capacitive cell constant was similar, large deviation from the theoretical line was observed.

C. Electrode area. Fig. 9 shows Bode plots of total impedance against frequency as the variation in the electrode

Table 2 Parameters for the circuit elements evaluated by fitting the impedance data using model II ( $D=$ variable, $W=20 \mu \mathrm{m})$

\begin{tabular}{llll}
\hline$(D, W)$ & $C_{\text {Cell }} / \mathrm{pF}$ in air & $C_{\text {Cell }} / \mathrm{pF}$ in DW & $R_{\text {Sol }} / \mathrm{M} \Omega$ in DW \\
\hline$(20,20)$ & $0.311 \pm 0.005$ & $0.413 \pm 0.009$ & $5.43 \pm 0.115$ \\
$(100,20)$ & $0.295 \pm 0.007$ & $0.355 \pm 0.007$ & $21.0 \pm 0.624$ \\
$(500,20)$ & $0.288 \pm 0.004$ & $0.342 \pm 0.006$ & $90.2 \pm 2.37$ \\
\hline
\end{tabular}

Table 3 Capacitance magnitude at $10 \mathrm{~Hz}$ and phase angle extracted by the simulation of CPE component $(D=$ variable, $W=20 \mu \mathrm{m})$

\begin{tabular}{llll}
\hline$(D, W)$ & $C_{\text {Int }} / \mathrm{nF}$ & $C_{\text {Char }} / \mathrm{F} \mathrm{m}^{-2}$ & Phase angle \\
\hline$(20,20)$ & $0.510 \pm 0.023$ & $0.255 \pm 0.011$ & $64.91 \pm 0.82$ \\
$(100,20)$ & $0.320 \pm 0.014$ & $0.160 \pm 0.007$ & $76.03 \pm 1.33$ \\
$(500,20)$ & $0.275 \pm 0.016$ & $0.138 \pm 0.008$ & $75.09 \pm 1.39$ \\
\hline
\end{tabular}


(a)

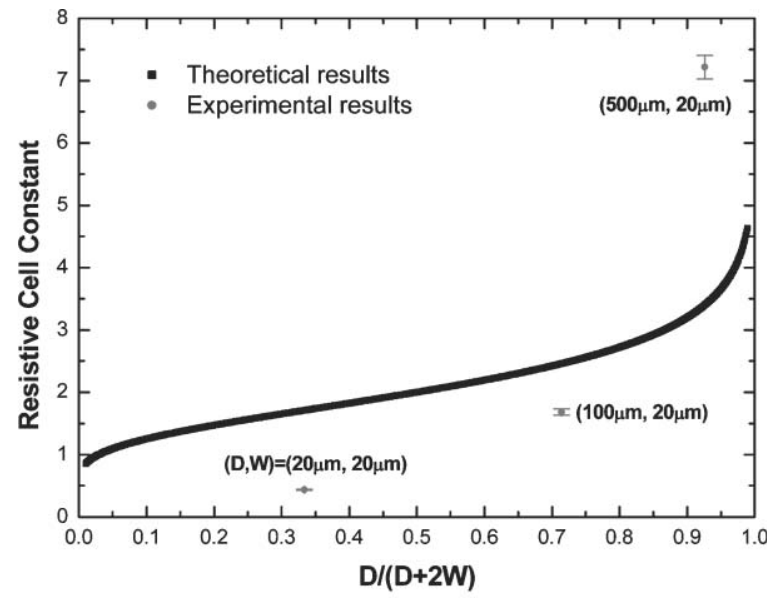

(b)

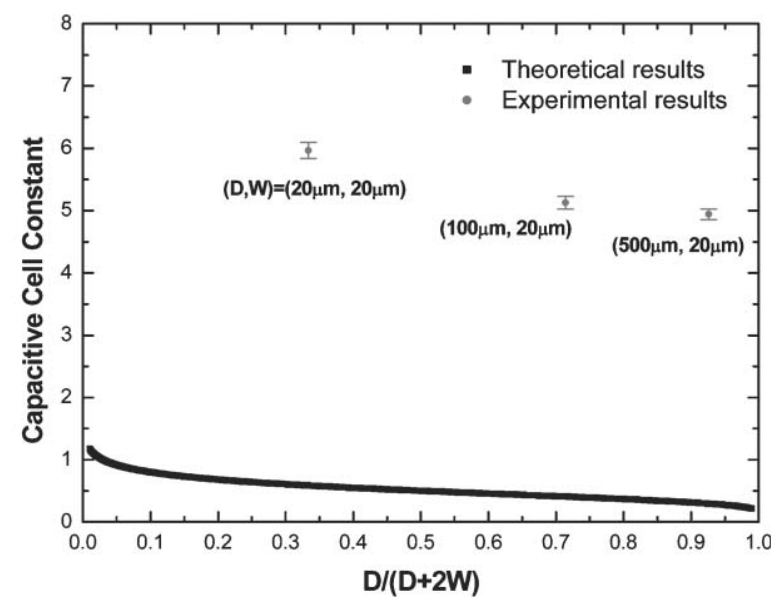

Fig. 8 Comparisons between the experimentally obtained cell constants and the theoretically predicted values as the change of interelectrode spacing: (a) resistive cell constant and (b) capacitive cell constant.

width. As the electrode area increased, both $f_{\text {high }}$ and total impedance were not changed so much but $f_{\text {low }}$ decreased significantly. Table 4 lists in fitting results, $C_{\text {Cell }}$ and $R_{\text {Sol }}$, as a function of electrode width $(D=100 \mu \mathrm{m})$. As the electrode area increased, $C_{\text {Cell }}$ increased slightly, but $R_{\text {Sol }}$ was not changed so much. Table 5 lists in the extracted data of capacitance magnitude and phase angle from CPE component as the variation in electrode width. The magnitude increased with the increase in electrode area. But we also observed the decrease in phase angle. We think that the change in $f_{\text {low }}$ should result from the increase in $C_{\mathrm{DL}}$. Therefore, we confirmed that the shift of cut-off frequencies observed above should accord with theoretical considerations in section 3.1.

Fig. 10 shows the comparisons between measured cell constants and theoretically obtained values. Theoretically, as the electrode width increases, the resistive cell constant decreases but the capacitive one increases. We observed that the measured resistive cell constants were not changed with the increase in $W$. Although the capacitive ones showed a similar manner, a large deviation from the theoretical curve existed. (a)

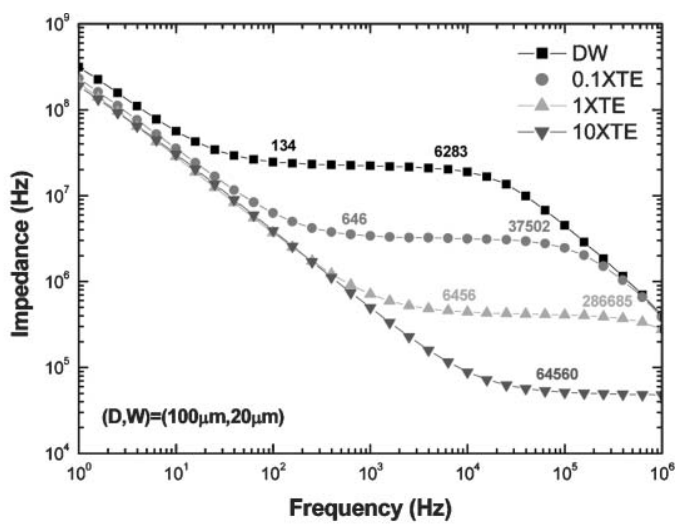

(b)

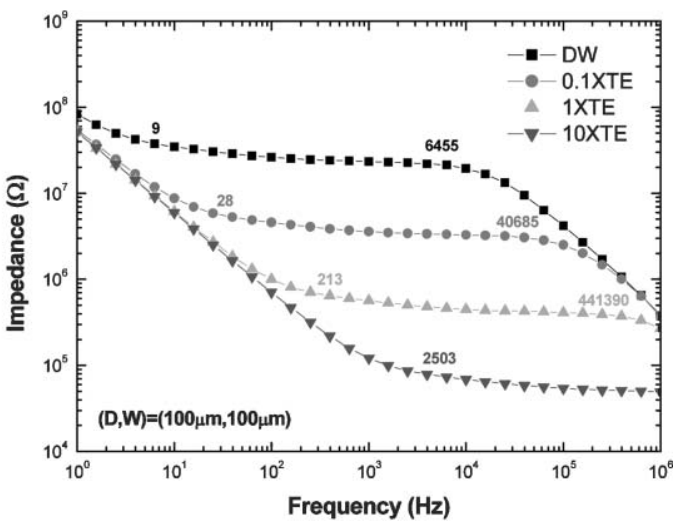

(c)

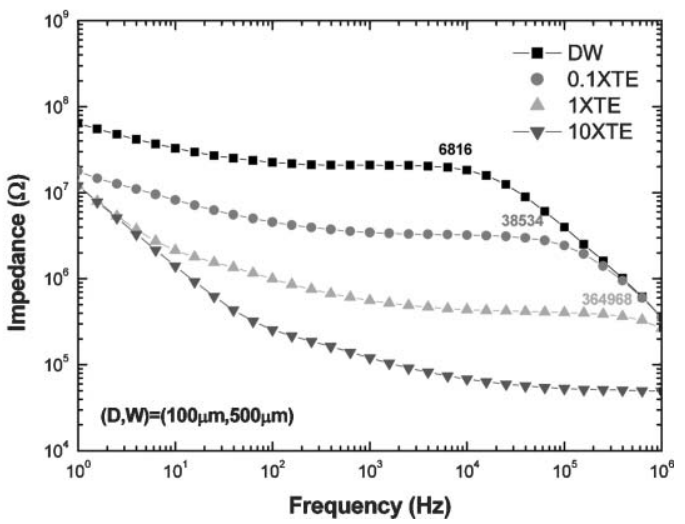

Fig. 9 Bode plots of total impedance against frequency as the variation in the electrode width $(D=100 \mu \mathrm{m})$

Table 4 Parameters for the circuit elements evaluated by fitting the impedance data using model II ( $D=100 \mu \mathrm{m}, W=$ variable $)$

\begin{tabular}{llll}
\hline$(D, W)$ & $C_{\text {Cell }} / \mathrm{pF}$ in Air & $C_{\text {Cell }} / \mathrm{pF}$ in DW & $R_{\text {Sol }} / \mathrm{M} \Omega$ in DW \\
\hline$(100,20)$ & $0.295 \pm 0.007$ & $0.355 \pm 0.007$ & $21.0 \pm 0.624$ \\
$(100,100)$ & $0.316 \pm 0.004$ & $0.381 \pm 0.006$ & $21.5 \pm 0.559$ \\
$(100,500)$ & $0.339 \pm 0.004$ & $0.403 \pm 0.005$ & $20.9 \pm 0.351$ \\
\hline
\end{tabular}

Table 5 Capacitance magnitude at $10 \mathrm{~Hz}$ and phase angle extracted by the simulation of CPE component ( $D=100 \mu \mathrm{m}, W=$ variable)

\begin{tabular}{llll}
\hline$(D, W)$ & $C / \mathrm{nF}$ & $C_{\text {Char }} / \mathrm{F} \mathrm{m}^{-2}$ & Phase angle \\
\hline$(100,20)$ & $0.320 \pm 0.014$ & $0.160 \pm 0.007$ & $76.02 \pm 0.89$ \\
$(100,100)$ & $1.222 \pm 0.117$ & $0.122 \pm 0.012$ & $55.20 \pm 2.10$ \\
$(100,500)$ & $1.522 \pm 0.104$ & $0.030 \pm 0.021$ & $45.40 \pm 1.64$ \\
\hline
\end{tabular}


(a)

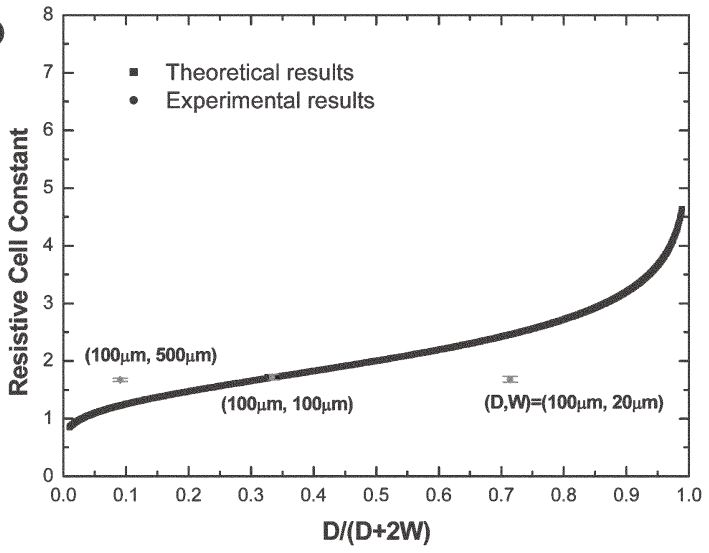

(b)

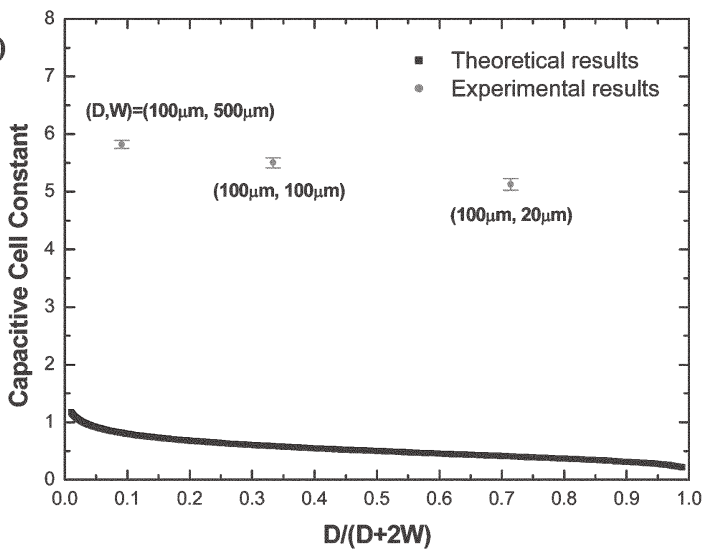

Fig. 10 Comparisons between the experimentally obtained cell constants and the theoretically predicted values with change of electrode width: (a) resistive cell constant and (b) capacitive cell constant.

D. Sources of deviation. We assumed a uniform current-line distribution between electrodes to calculate the cell constants simply. In the real situation, however, considerable spreading of the current lines occurs, and it is called the fringing effect. If a coplanar structure is used, the fringing effect is even more explicitly present. It is exclusively dependent on the electrode configuration and can therefore be calculated from the electrode geometry. Fig. 11 shows the steady-state results of conduction simulation of coplanar sensors placed into a microchannel using dedicated finite element programs. The color map indicates the electric field calculated from the electric potential. We can predict that the sensing field is converged on inner parts of sensors from this simulaton. We infer that the fringing effect between coplanar impedance sensors can be the factor to elucidate the disagreement between predictions and observed results in the resistive cell constant. Also, we speculate that the nano-scale side of coplanar sensors due to the film thickness cannot be ignored. Therefore, we can predict that the resistive cell constant increases steeply with an increase in $D$ and it is not changed with an increase in W.

However, the capacitance between coplanar electrodes varies from the resistance in some points. The coplanar electrodes are embedded in Pyrex glass and the only small area of electrodes contacts with solutions for sensing. Thus, the dielectric constant of Pyrex glass cannot be ignored in

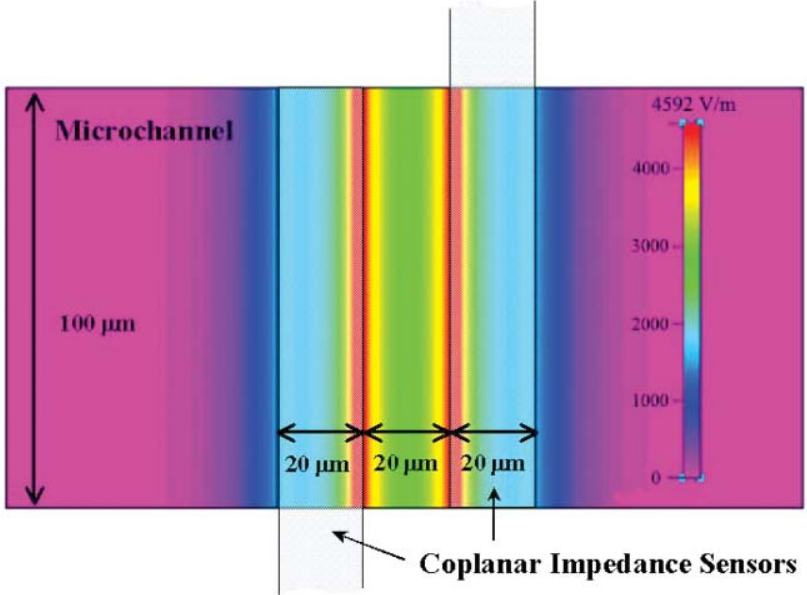

Fig. 11 Steady-state results of electric field calculated from the electric potential.

determining the cell capacitance. The capacitance of coplanar strips embedded in multilayer iso/anisotropic dielectrics have been studied in the field of MIC, electrooptic modulator and surface acoustic-wave application. ${ }^{38}$ To inquire further will lead us into the specialized area of transmission lines and coplanar strips, and takes us beyond the scope of this article. Briefly, the effect of the dielectric constant of Pyrex glass was investigated as follows. The capacitive coupling between coplanar electrodes can be approximated by the following formula for two parallel strip conductors on a dielectric substrate with a finite thickness: ${ }^{39}$

$$
C=\frac{\pi \varepsilon_{0} \varepsilon_{\mathrm{eff}} L_{\mathrm{T}}}{\left[\ln \left(\frac{\pi D}{W+t}+1\right)\right]}
$$

where $t$ is the vertical distance to the ground plate. The effective dielectric constant, $\varepsilon_{\text {eff }}$, is approximately $\varepsilon_{1 \mathrm{r}}$ if $D \gg t$ or $\varepsilon_{\text {eff }} \approx\left(\varepsilon_{1 \mathrm{r}}+\varepsilon_{2 \mathrm{r}}\right) / 2$ if $D \approx t$ (in this study, $\varepsilon_{1 \mathrm{r}}$ is 78.2 of water and $\varepsilon_{2 \mathrm{r}}$ is 4.6 of Pyrex 7740 glass). The capacitance of coplanar structure is plotted in Fig. 12 as a function of $D$ for a fixed value of $W$. Superimposed on this graph is the variation of capacitance as a function of $W$ for a fixed value of $D . C_{\text {Cell }}$

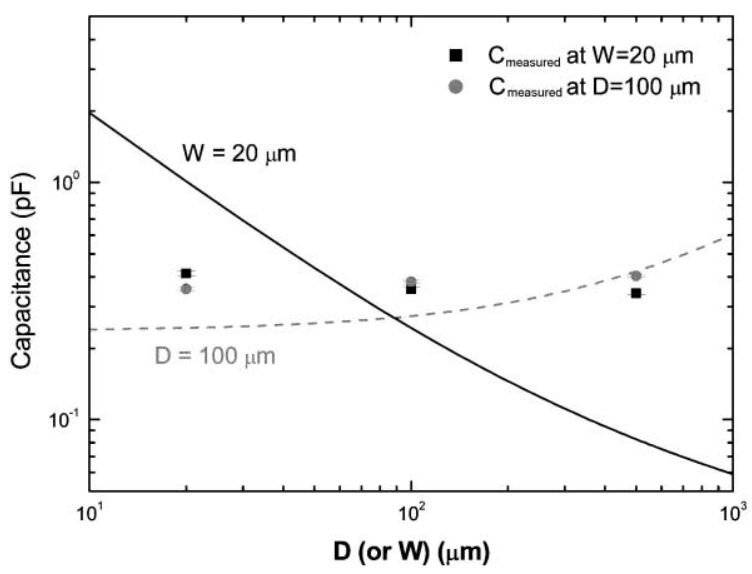

Fig. 12 Capacitance of coplanar structure as a function of interelectrode spacing (straight line) and electrode width (dashed line). 
decreases with an increase in $D$, and it increases with an increase in $W$. The deviation of measured data from the theoretical curves results from the assumption made in eqn. (13), the infinite thickness of media 1 (water in this study) since the water is filled in a microchannel with width $100 \mu \mathrm{m}$ and depth $30 \mu \mathrm{m}$. The parasitic capacitance due to the electrodes embedded in Pyrex glass is added to the capacitance of the sensing part.

We will shift the emphasis away from high-frequency values $\left(R_{\mathrm{Sol}}\right.$ and $\left.C_{\text {Cell }}\right)$ to interfacial capacitance as design parameters. We considered the impedance of the platinum thin-film electrode being in contact with an electrolyte such as CPE. Considerable effort has been made to understand the origin of the CPE in electrochemistry. ${ }^{40-44}$ Possible causes of the CPE are known to be of geometric origin: surface inhomogeneity and surface irregularity. The surface irregularity gives rise to the electrolytic resistive distribution, while the surface inhomogeneity brings about the capacitive distribution. An irregular and porous electrode geometry causes current density inhomogeneities and thus yields deviations from ideal capacitive behavior. But the surface inhomogeneity (interfacial capacitance inhomogeneity) results from a non-uniform surface charge density on the rough electrodes. Not only the difference in value but also the distribution of the capacitance are the important factors influencing capacitive distribution. At the same electrode area, a capacitance per unit area $\left(C_{\mathrm{Char}}\right)$ correlated inversely with phase angle as the variation of the interelectrode spacing. On the contrary, at the same spacing, $C_{\text {Char }}$ correlated directly with the phase angle as that of the electrode area. We speculate that the former results from the surface inhomogeneity while the latter results from the surface irregularity. That is, the interelectrode spacing is related to the surface inhomogeneity, and the electrode area is related to the surface irregularity in this study.

\subsection{Design rule of coplanar impedance sensors}

From the frequency characteristics of coplanar impedance sensors (Fig. 4), three types of components $\left(C_{\mathrm{DL}}, R_{\mathrm{Sol}}\right.$ and $\left.C_{\text {Cell }}\right)$ can be used as the signal generation for sensing biomolecules. At present, these sensors have been implanted on the microchip format, but the sensing mechanism is just limited to the change of conductivity related to $R_{\mathrm{Sol}}$. Furthermore, few researchers were concerned about the correlation between design parameters and frequency behavior in coplanar impedance sensors.

A. Interfacial capacitance ( $\boldsymbol{C}_{\mathrm{DL}}$ (or $\left.\mathrm{CPE}\right)$ ). The interfacial capacitance (of the level $\mathrm{nF}$ to $\mu \mathrm{F}$ ) can be used as a sensing mechanism combined with specific surface reactions. When target DNA hybridizes to oligonucleotide probes or when antibodies bind to antigens, the change in electric properties results in a change of impedance, enabling the measurement of direct electrical signal, such as magnitude and phase angle. The surface modification may be accompanied to improve the sensitivity and selectivity (i.e. an antigen-antibody layer is the order of $10-100 \mathrm{~nm}$ and a probe-DNA binding is up to $200 \mathrm{~nm}$ ). The interfacial capacitance is the electric property in the low-frequency region, and so the low cut-off frequency will be increased to enlarge the range of working frequencies. The increase in a low cut-off frequency results from the decrease in interelectrode spacing and electrode width. However, the decrease in electrode area may result in the decrease in the magnitude of interfacial capacitance. The interdigitated electrode arrays will be needed to amplify the signal since the multiplication of the area of individual electrodes increases the total capacitance.

B. Solution resistance $\left(\boldsymbol{R}_{\text {Sol }}\right)$. The change in electrolyte conductivity is commonly adopted as the sensing mechanism of on-chip based coplanar impedance sensors. Normally, the electrolyte conductivity detection is a non-specific measurement method except when combined with capillary electrophoresis where specific ions are separated prior to the detection. In this conductivity detection, it is important that the frequency band between the low and high cut-off frequencies should be as large as possible. The width of frequency band can be expressed as:

$$
\text { Band width }=\log \frac{f_{\text {high }}}{f_{\text {low }}} \approx \frac{C_{\mathrm{DL}}}{C_{\text {Cell }}}
$$

The increase in electrode area will result in the increase in the width of frequency band. The interelectrode spacing, which is related to capacitive cell constant, has an influence on the frequency band. But the electrode area is a more sensitive parameter than the interelectrode distance in coplanar impedance sensors. On the other hand, the sensitivity of detection (amount of ionic charge detectable) is related to the resistive cell constant. To increase the sensitivity of detection in this conductivity mode, the resistive cell constant, which was sensitive to the interelectrode distance, must be increased. However, the submicron electrode gap and size will not be suitable to improve the sensitivity of the coplanar impedance sensors.

C. Cell capacitance $\left(C_{\text {Cell }}\right)$. The change in dielectric constant of the solution can be used as a sensing mechanism although the working frequency is high. The coplanar electrodes are usually embedded in dielectric substrates (e.g. glass, quartz and polymer) and the only small area of electrodes contacts with biomolecule solutions for sensing. The parasitic capacitance due to dielectric substrates cannot be ignored. Thus, the parasitic capacitance must be reduced to enhance the sensitivity. Theoretically, the capacitance of coplanar strips is sensitive to the change of interelectrode distance compared with electrode width. Moreover, because total capacitance is a level of $\mathrm{pF}$ in microscale, this detection using the change of dielectric constant will have the limitation of sensing biomolecules.

\section{Concluding remarks}

Glass-based microchannel chips were fabricated using photolithographic technology, and Pt thin-film microelectrodes, as coplanar impedance sensors, were integrated on them. Longitudinal design parameters, such as interelectrode spacing and electrode width, of coplanar impedance sensors were changed to determine AC frequency characteristics as design 
parameters. Through developing total impedance equations and modeling equivalent circuits, the dominant components in each frequency region were illustrated for coplanar impedance sensors and measured results were compared with fitted values. As the ionic concentration increased, the value of frequencyindependent region decreased and cut-off frequencies increased. As the interelectrode spacing increased, cut-off frequencies decreased and total impedance increased. However, the width of each frequency-independent region was similar. As the electrode area increased, $f_{\text {low }}$ decreased but $f_{\text {high }}$ was fixed. We think that the decrease in $R_{\text {Sol }}$ dominated over the influence of other components, which resulted in heightening $f_{\text {low }}$ and $f_{\text {high }}$. The interelectrode spacing is a more significant parameter than the electrode area in the frequency characteristics of coplanar sensors. The cell constant dependent on the geometry of the sensor was developed, and we compared the theoretically predicted values with the experimentally obtained results. The deviation of experimentally obtained results from theoretically predicted values may result from the fringing effect of coplanar electrode structure and parasitic capacitance due to dielectric substrates. We suggest the guidelines of dominant components, such as $C_{\mathrm{DL}}, R_{\mathrm{Sol}}$ and $C_{\text {Cell }}$, for sensing as design parameters.

\section{Acknowledgements}

We would like to thank Dr. Yoon Kyung Cho, Dr. Kwang Wook $\mathrm{Oh}$ and Youngsun Lee at the Samsung Advanced Institute of Technology (SAIT) for fruitful discussions during sample preparation and capacitance measurements. This work was supported by the Ministry of Education ("Brain Korea 21") and the Ministry of Science and Technology ("21C Frontier R\&D Program").

Jongin Hong, ${ }^{* a b}$ Dae Sung Yoon, ${ }^{* b}$ Sung Kwan Kim, ${ }^{a}$ Tae Song Kim, ${ }^{b}$ Sanghyo Kim, ${ }^{c}$ Eugene Y. Pak ${ }^{c}$ and Kwangsoo No ${ }^{a}$

${ }^{a}$ Department of Material Science and Engineering, Korea Advanced Institute of Science and Technology, 373-1 Kuseong-dong, Yuseong-gu, Daejeon, Korea.E-mail: kairduck@kaist.ac.kr

${ }^{b}$ Bio-MEMS, Microsystem Research Center, Korea Institute of Science and Technology, PO Box 131, Cheongryang, Seoul, Korea.

E-mail:dsyoon@kist.re.kr

${ }^{c}$ BioMEMS, Digital Bio Lab., Samsung Advanced Institute of

Technology, Suwon, Korea

\section{References}

1 A. Manz, N. Graber and H. M. Widmer, Sens. Actuators B, 1990, 1, 244-248.

2 P.-A. Auroux, D. Lossifidis, D. R. Reyes and A. Manz, Anal. Chem., 2002, 74, 2637-2652.

3 J. C. Fister, S. C. Jacobson, L. M. Davis and J. M. Ramsey, Anal. Chem., 1998, 70, 431-437.

4 B. B. Haab and R. A. Matheis, Anal. Chem., 1999, 71, 5137-5145.

5 J. Wang, Anal. Chem., 1999, 71, 328R-332R.

6 E. Bakker and M. Telting-Diaz, Anal. Chem., 2002, 74, 2781-2800.

7 M. A. Schwarz and P. C. Hauser, Lab Chip, 2001, 1, 1-6.

8 A. Bossi, S. A. Piletsky, P. G. Righetti and A. P. F. Turner, J. Chromatogr., A, 2000, 892, 143-153.

9 N. A. Lacher, K. E. Garrison, R. S. Martin and S. M. Lunte, Electrophoresis, 2001, 22, 2526-2536.

10 B. Saif, R. K. Mohr, C. J. Montrose and T. A. Litovitz, Biopolymer, 1991, 31, 1171-1180.
11 M. S. DeSilva, Y. Zhang, P. J. Hesketh, G. J. Maclay, S. M. Gendel and J. R. Stetter, Biosens. Bioelectron., 1995, 10, 675-682.

12 H. Berney, J. Alderman, W. Lane and J. K. Collins, Sens. Actuators, B, 1997, 44, 578-584.

13 V. M. Mirsky, M. Riepl and O. S. Wolfbeis, Biosens. Bioelectron., 1997, 12, 9-10, 977-989.

14 H. E. Ayliffe, A. B. Frazier and R. D. Rabbit, IEEE J. Microelectromech. Syst., 8, 1, 50-57.

15 M. Riepl, V. M. Mirsky, I. Novotny, V. Tvarozek, Vlastimil Rehacek and O. S. Wolfbeis, Anal. Chim. Acta, 1999, 392, 77-84.

16 W. Laureyn, D. Nelis, P. V. Gerwen, K. Baert, L. Hermans, R. Magnée, J.-J. Pireaux and G. Maes, Sens. Actuators, B, 2000, 68, $360-370$

17 M. J. Schönig, A. Kurowski, M. Thust, P. Kordos, J. W. Schultze and H. Lüth, Sens. Actuators, B, 2000, 64, 59-64.

18 R. Gomez, R. Bashir, A. Sarikaya, M. R. Ladisch, J. Sturgis, J. P. Robinson, T. Geng, A. K. Bhunia, H. L. Apple and S. Wereley, Biomed. Microdev., 2001, 3, 3, 201-209.

19 B. Timmer, W. Sparreboom, W. Olthuis, P. Bergveld and A. van den Berg, Lab Chip, 2002, 2, 121-124.

20 L. Yang, Y. Li, C. L. Griffis and M. G. Johnson, Biosens. Bioelectron., 2004, 19, 1139-1147.

21 J. Hong, D. Yoon, M.-I. Park, J. Choi, T. Song, G. Im, S. Kim, Y. Park and K. No, Jpn. J. Appl. Phys., 2004, 43, 8A, 5639-5645.

22 B.-W. Chang, C.-H. Chen, S.-J. Ding, D. C.-H. Chen and H.-C. Chang, Sens. Actuators, B, 2004, in press.

23 M. Galloway and S. A. Soper, Electrophoresis, 2002, 23, 3760-3768.

24 M. Galloway, W. Stryjewski, A. Henry, S. M. Ford, S. Llopis, R. L. McCarley and S. A. Soper, Anal. Chem., 2002, 74, $2407-2415$.

25 A. Berthold, F. Laugere, H. Schellevis, C. R. de Boer, M. Laros, R. M. Guijt, P. M. Sarro and M. J. Vellekoop, Electrophoresis, 2002, 23, 3511-3519.

26 J. Lichtenberg, F. de Rooij Nico and E. Verpoorte, Electrophoresis, 2002, 23, 3769-3780.

27 A. J. Zemann, Electrophoresis, 2003, 24, 2125-2137.

28 F. Laugere, R. M. Gujit, J. Bastemeijer, Gert van der Steen, Axel Berthold, E. Baltussen, P. Sarro, G. W. K. van Dedem, M. Vellekoop and A. Bossche, Anal. Chem., 2003, 75, 306-312.

29 P. Jacobs, A. Varlan and W. Sansen, Med. Biol. Eng. Comput., 1995, 33, 802-810.

30 M. J. Madou, Fundamentals of Microfabrication: The Science of Minaturization, 2nd edn., CRC Press, London, 2002.

31 M. Stjernström and J. Roeraade, J. Micromech. Microeng., 1998, 8, 33-38.

32 S.-I. Pyun, Outlines of Electrochemistry of Materials, Sigma Press, 2001.

33 A. Bard and L. Faulkner, Electrochemical Methods: Fundamentals and Applications, John Wiley \& Sons, New York, 1980.

34 R. J. Hunter, Zeta Potential in Colloid Science, Academic Press, London, 1981.

35 V. S. Muralidharan, Anti-Corrosion Methods Mater., 1997, 44, 1, 26-29.

36 A. Dabrowska and W. Wieczorek, Mater. Sci. Eng. B, 1994, 22, 107-116.

37 K. J. Binns and P. J. Lawrenson, "Analysis and Computation of Electric and Magnetic Field Problems", 2nd Ed., Pergamon Press, New York, (1973).

38 S. K. Koul and B. Bhat, IEEE Trans. Microwave Theory Tech., 1983, MTT-31, 12, 1051-1059.

39 L. K. Baxter, Capacitive Sensors: Design and Applications, IEEE Press, New York, 1997.

40 L. I. Daikhin, Phys. Rev. E, 1996, 53, 6, 6192-6199.

41 L. I. Daikhin, A. A. Kornyshev and M. Urhakh, Electrochim. Acta, 1997, 42, 19, 2853-2860.

42 E. Lust, A. Jänes, V. Sammelselg and P. Miidla, Electrochim. Acta, 2000, 46, 185-191.

43 Z. Kerner and T. Pajkossy, Electrochim. Acta, 2000, 46, 207-211.

44 C.-H. Lee, S.-I. Pyun and J.-H. Kim, Electrochim. Acta, 2003, 48, $3455-3463$. 